\title{
Necessidades de saúde das pessoas com tuberculose pulmonar*
}

Health needs of people with pulmonary tuberculosis

Necesidades de salud de las personas con tuberculosis pulmonar

\section{Aliéren Honório Oliveira ${ }^{\mathrm{I}}$, Antonio Germane Alves Pinto ${ }^{\mathrm{II}}$, Glauberto da Silva Quirino ${ }^{\mathrm{III}}$ Rachel de Sá Barreto Luna Callou Cruz ${ }^{\mathrm{IV}}$, Maria Lúcia Duarte Pereirav , Edilma Gomes Rocha Cavalcantevi}

\begin{abstract}
Resumo: Objetivo: analisar as necessidades de saúde das pessoas em tratamento para a tuberculose assistidas pelos serviços de saúde. Método: estudo descritivo, com abordagem qualitativa, realizado com dez pacientes assistidos pelas equipes da Estratégia Saúde da Família e serviço de referência. As entrevistas foram submetidas à técnica de análise temática e analisadas conforme a Taxonomia Operacional de Necessidades de Saúde. Resultados: os pacientes relataram necessidades de alimentação e moradia, mostraram satisfeitos com o tratamento, acessibilidade e cordialidade dos profissionais de saúde, mas insatisfeitos com a investigação dos sintomas e evolução do quadro clínico. O vínculo com o enfermeiro foi positivo. Orientações e busca de informações subsidiaram a autonomia e o modo de andar a vida. Conclusão: os pacientes reconheceram as necessidades de saúde referentes ao acesso às tecnologias que melhoram e prolongam a vida, mas requererem investigação diagnóstica precisa, orientação para autonomia e ordenação da Atenção Primária à Saúde.

Descritores: Tuberculose; Atenção à Saúde; Acolhimento; Autonomia Pessoal; Integralidade em Saúde
\end{abstract}

\footnotetext{
I Enfermeira. Mestre em Enfermagem. Enfermeira da Secretaria de Saúde do Município de Farias Brito, CE, Brasil. E-mail: alierenoliveira@hotmail.com. Orcid: http://orcid.org/0000-0003-4702-3691

II Enfermeiro. Doutor em Saúde Coletiva. Docente do Departamento de Enfermagem da Universidade Regional do Cariri, Programa de PósGraduação em Enfermagem, Crato, CE, Brasil. E-mail: germanepinto@hotmail.com. Orcid: http://orcid.org/0000-0002-4897-1178

III Enfermeiro. Doutor em Educação em Ciências: Química da Vida e Saúde. Docente do Departamento de Enfermagem da Universidade Regional do Cariri, Programa de Pós-Graduação em Enfermagem, Crato, CE, Brasil; E-mail: glauberto.quirino@urca.br. Orcid: https://orcid.org/0000-0001-5488-7071

IV Enfermeira. Doutora em Saúde Materno Infantil. Docente do Departamento de Enfermagem da Universidade Regional do Cariri, Programa de Pós-Graduação em Enfermagem, Crato, CE, Brasil. E-mail: rachel.callou@hotmail.com. Orcid: http://orcid.org/0000-0002-4596-313X.

V Enfermeira. Doutora em Enfermagem. Docente do Departamento de Enfermagem da Universidade Estadual do Ceará, Fortaleza, CE, Brasil; E-mail: luciad029@gmail.com. Orcid: http://orcid.org/0000-0002-7685-6169.

VI Enfermeira. Doutora em Ciência. Docente do Departamento de Enfermagem da Universidade Regional do Cariri, Programa de PósGraduação em Enfermagem, Crato, CE, Brasil. E-mail: edilma.rocha@yahoo.com.br, Orcid: http://orcid.org/0000-0002-6861-2383
}

* Extraído da dissertação "Necessidades de saúde das pessoas com tuberculose na construção do cuidado integral”, Programa de Pós-Graduação em Enfermagem da Universidade Regional do Cariri (URCA), 2016. 


\begin{abstract}
Objective: to analyze the health needs of people undergoing treatment for tuberculosis assisted by health services. Method: a descriptive study with a qualitative approach, carried out with ten patients assisted by the Family Health Strategy teams and a reference service. The interviews were submitted to the thematic analysis technique and analyzed according to the Operational Taxonomy of Health Needs. Results: the patients reported needs for food and housing, were satisfied with the treatment, accessibility and cordiality of the health professionals, but dissatisfied with the investigation symptoms and evolution of the clinical picture. The bond with the nurse was positive. Orientations and search for information subsidized the autonomy and the way of living life. Conclusion: patients recognized the health needs related to access to technologies that improve and prolong life, but require precise diagnostic investigation, guidance for autonomy and ordering Primary Health Care.
\end{abstract}

Descriptors: Tuberculosis; Health Care; User Embracement; Personal Autonomy; Integrality in Health

Resumen: Objetivo: analizar las necesidades de salud de personas en tratamiento por tuberculosis asistidas por servicios de salud. Método: estudio descriptivo, cualitativo, realizado con diez pacientes asistidos por los equipos de Estrategia Salud de la Familia y servicio de referencia. Entrevistas sometidas a la técnica de análisis temático y analizadas según la Taxonomía Operativa de Necesidades de Salud. Resultados: los pacientes reportaron necesidades de alimentación y residencia, se mostraron satisfechos con el tratamiento, accesibilidad y cordialidad de los profesionales, pero insatisfechos con la investigación sobre los síntomas y evolución del cuadro clínico. El vínculo con el enfermero fue positivo. Orientaciones y búsqueda de información subvencionaban la autonomía y la forma de vivir. Conclusión: los pacientes reconocieron las necesidades de salud relacionadas con el acceso a tecnologías que mejoran y prolongan la vida, pero requieren una investigación diagnóstica precisa, orientación para la autonomía y ordenamiento de la Atención Primaria de Salud.

Descriptores: Tuberculosis; Atención a la Salud; Acogimiento; Autonomía Personal; Integralidad en Salud

\title{
Introdução
}

A tuberculose (TB) ainda se apresenta como problema de saúde pública que requer investimento em ações rápidas para a cobertura universal de saúde, que permita o acesso ao serviço de atendimento à saúde, sem ônus financeiro, na atenção primária. Além da proteção social ao se considerar os determinantes sociais das pessoas doentes que têm impacto sobre o agravo. ${ }^{1}$

A relação entre as desigualdades sociais e a TB aponta articulação com a dimensão social da população, o que envolve idade, renda, desemprego, acesso aos serviços de saúde, condições de vida e trabalho. Esses fatores potencializam a produção e a reprodução social dos determinantes da doença que dificultam o seu controle e requerem a organização da sociedade para ampliar as estratégias de enfrentamento. ${ }^{2}$ Assim, as desigualdades sociais apresentam-se como desafios para o controle da TB quando atingem populações menos favorecidas, especialmente as desprovidas de condições materais cuja vulnerabilidade ao adoecimento e a 
internação são marcadas em histórias que apontam a ausência de proteção aos direitos humanos. ${ }^{3}$ Tais condições de vida das pessoas com TB demandarão maiores esforços por parte dos profissionais de saúde que devem considerar, escutar e atender as suas necessidades de saúde.

Nesse sentido, adotou-se no presente estudo a Taxonomia Operacional de Necessidades de Saúde, que tem como conclusão teórica a dimensão social dos fenômenos da saúde com condições particulares. Essas necesidades são sempre históricas, dinâmicas e cambiantes, com componente de natureza subjetiva e individual. Apontam como perspectiva repensar a equidade, a integralidade, a reogranização e a operacionalidade das práticas de saúde. ${ }^{4}$

A taxonomia resulta de quatro elementos. O primeiro refere-se à necessidade de boas condições de vida que define a saúde como conjunto de possibilidade dado em uma certa sociedade; de qualidade de vida que visa superar a visão polarizada da medicina e da adoção de recursos para garantir a atenção integral à saúde. Enquanto a doença é definida como um sinal de existência privada e expressa a dificuldade na vida de uma pessoa com condições desfavoráveis (acesso às condições de moradia e hábitos pessoais). ${ }^{4}$

O segundo relaciona-se à necessidade de garantia de acesso a todas as tecnologias que melhorem e prolonguem a vida. Ele aponta a demanda individual e coletiva na procura de acesso a tecnologias leves, leve-duras e duras que os serviços de saúde devem oferecer e traduz as aspirações da sociedade por novos padrões de direitos sociais (direito à vida saudável, ao alívio da dor, uma velhice ativa e prazerosa). ${ }^{4}$

O terceiro elemento versa sobre a necessidade de ter vínculo com um profissional da equipe (sujeito em relação), com a construção de espaços propícios de vínculo afetivo, vínculo doente/família e vínculo como aspecto essencial da prática clínica, sendo o paciente capaz de potencializar transformações nessa prática. ${ }^{4}$

Por fim, a necessidade de autonomia e autocuidado na escolha do "modo de andar a vida" (a construção do sujeito) pressupõe demonstrar que a passividade é um empecilho à cura, à 
prevenção e à promoção da saúde. Assim sendo, o paciente deve ampliar sua capacidade de entender seu próprio corpo, doença, sua relação com o meio social e de sobrevivência, a vida e a qualidade de vida. Além de incorporar ideias do pensamento crítico em Educação em Saúde, que são partes do processo de construção de autonomia de cada pessoa. ${ }^{4}$

Desse modo, o enfermeiro da Atenção Primária à Saúde tem utilizado fatores pessoais e ambientais articulados ao uso de tecnologias em saúde, que são relativas ao acolhimento, à comunicação e ao vínculo no decorrer do tratamento, usados como ferramentas para desenvolver cuidado estruturado com ações necessárias ao sucesso do tratamento da TB e redução das fragilidades por meio de ações preventivas. ${ }^{5}$ Além disso, devem utilizar práticas centradas no usuário, como o tratamento diretamente observado. ${ }^{6}$ Ressalta-se que, para isso, os profissionais de saúde precisam considerar o cenário sociocultural e psicossocial do adoecimento da pessoa com TB, o que permite compreender como constroem o seu cotidiano e têm autonomia para modificá-lo ao articular conhecimento e práticas protetoras. ${ }^{3 .}$

Em geral, as pessoas com TB são afetadas negativamente tanto no ambiente doméstico quanto no social, necessitando de ajuste psicossocial à doença e de apoio diante do risco de adaptarem-se mal ao adoecimento. ${ }^{7}$ Essa situação repercute no autocuidado e na tomada de decisões quanto à busca de melhores condições de vida.

Nesse contexto, o desenvolvimento do presente estudo justifica-se pelas necessidades em saúde comuns às pessoas com $\mathrm{TB}$, que podem ser identificadas no acesso às tecnologias, no vínculo com a equipe na informação em saúde para sua autonomia e autocuidado. Essas dimensões determinam a relevância do tema diante das desigualdades de saúde e da possibilidade de construção de um cuidado integral pelos profissionais de saúde. Esta pesquisa objetivou analisar as necessidades de saúde das pessoas em tratamento para a TB assistidas pelos serviços de saúde. 
5 | Oliveira AH, Pinto AGA, Quirino GS, Cruz RSBL, Pereira MLD, Cavalcante EGR

\section{Método}

Trata-se de estudo exploratório, com abordagem qualitativa, baseado nos elementos da Taxonomia Operacional de Necessidades de Saúde, que compreende necessidade de boas condições de vida; necessidade de garantia de acesso a todas as tecnologias que melhorem e prolonguem a vida; necessidade de ter vínculo com um profissional da equipe; necessidade de autonomia e autocuidado na escolha do "modo de andar a vida".

O município, localizado na região sul do estado do Ceará, apresentava um total de 40 equipes da Estratégia Saúde da Família (ESF) e um centro de especialidade. Esse último contava com médico infectologista, enfermeiro e fisioterapeuta para garantir a assistência aos pacientes com doenças infecciosas. Os pacientes com TB tinham acesso a esse serviço por meio de referência ou demanda espontânea.

Nesses serviços de saúde, identificaram-se 25 casos de pessoas com TB pulmonar. Elegeram-se dez participantes, sendo oito atendidos pela equipe da ESF (zona urbana e zona rural) e dois pacientes do centro de especialidade que atenderam aos critérios de inclusão.

Foram critérios de inclusão: ter idade mínima de 18 anos; diagnóstico de TB pulmonar; ser acompanhado pela equipe da ESF/centro de especialidade; e encontrar-se na fase de manutenção do tratamento. Esse período justifica-se por acreditar-se que o tempo percorrido do tratamento é importante para que o indivíduo possa sentir alguma necessidade de saúde. Excluiu-se um paciente após mudança de diagnóstico durante o período da coleta de dados. Houve duas recusas em participar da pesquisa, quatro abandonaram o tratamento, cinco tiveram alta e três excluídos por não atenderem aos critérios de inclusão.

Para a coleta de dados, realizada no período de março a junho de 2015, utilizou-se entrevista semiestruturada, que contemplou questões norteadas sobre as necessidades de saúde que buscaram identificar significados sobre a saúde e estar doente pela TB e suas necessidades de saúde; quanto aos serviços de saúde que tiveram acesso, exames e recursos que contribuíram 
para o tratamento; a relação estabelecida entre os profissionais de saúde e a escolha do local de tratamento; as orientações recebidas durante doença/tratamento; e o acesso à informação e à participação de grupo de educação em saúde. Realizou-se contato face a face e as entrevistas ocorreram na unidade de saúde ou domicílio, sendo gravadas, com duração média de 35 minutos, e, posteriormente, transcritas na íntegra.

Para material empírico, utilizou-se a técnica de análise temática, que se pauta na descoberta dos núcleos de sentido que estejam presentes e frequentes nos significados do objeto de estudo. A técnica é executada por etapas de pré-análise, com leitura flutuante e apreensão das ideias centrais transmitidas nos dados; constituição do corpus representativos do material; e a reformulação dos objetivos analíticos pela releitura exaustiva e composição codificada do contexto e registros; em seguida, a exploração do material, com a compreensão do texto pelas formulações das categorias temáticas. Por fim, o tratamento dos resultados obtidos e interpretação com base na literatura. ${ }^{8}$ Os resultados foram descritivos e apresentados em quadros. As categorias analíticas foram baseadas nos elementos da Taxonomia Operacional de Necessidade de Saúde. ${ }^{4}$

O projeto de pesquisa foi apreciado e aprovado em 5 de março de 2015 pelo Comitê de Ética em Pesquisa (CEP) da Universidade Regional do Cariri, sob parecer 974.841 (CAAE: 42118915.4.0000.5055), e atendeu às Diretrizes e Normas Regulamentadoras de Pesquisas Envolvendo Seres Humanos (Resolução CNS 466/12). Aos participantes do estudo foi entregue um Termo de Consentimento Livre e Esclarecido, que foi assinado em duas vias (uma para o participante e outra para o pesquisador), autorizando a participação voluntária. Para garantir o anonimato, os participantes foram denominados como E1, E2 e assim por diante, conforme sequência de realização das entrevistas. 


\section{Resultados}

Nesta investigação, as pessoas com TB eram adultas, na faixa etária entre 20 e 65 anos (média de 40 anos de idade), declararam-se pardas, com baixo nível de renda (1 salário mínimo: $\mathrm{R} \$ 788,00)$ e escolaridade. Metade dos participantes encontrava-se fora do mercado formal de trabalho e duas famílias recebiam benefícios do governo (Bolsa Família), dividindo a renda com duas a oito pessoas em habitações precárias devido ao acúmulo de lixo e à ausência de saneamento básico.

\section{Necessidades de boas condições de vida}

O processo saúde-doença da TB foi entendido com concepções divergentes. Saúde representou qualidade de vida, independência, felicidade e fé; doença correspondeu à deteriorização, negação, dependência e tristeza relacionada à doença. A transmissão da TB foi associada às condições ambientais em que vivem relativas ao ar poluído, tempo frio, poeira e lixão, além do uso de álcool, tabagismo e processos gripais. As necessidades de saúde foram apontadas como melhores condições de vida, alimentação, trabalho, recursos financeiros, mudanças de hábitos e o autocuidado diante do processo de adoecimento.

Quadro 1 - Percepção dos pacientes com tuberculose em tratamento quanto às necessidades de boas condições de vida. Crato, CE, Brasil, 2015

\begin{tabular}{|c|c|}
\hline Saúde & $\begin{array}{r}\text { É melhor ter a sua saúde, ter sua força [...] é melhor a gente não ter nada, ter o poder de Deus e ter } \\
\text { saúde que nós vencemos qualquer batalha. (E4) }\end{array}$ \\
Com saúde a pessoa é tudo, e sem saúde a pessoa não é nada. (E3)
\end{tabular}




\section{Necessidade de garantia de acesso a todas as tecnologias que melhorem e prolonguem a vida}

Os pacientes procuraram o serviço de saúde apresentando tosse, hemoptise, febre, cansaço e emagrecimento. O diagnóstico ocorreu na unidade especializada, com tempo inferior a 1 mês e superior de mais de 1 ano. Foram porta de entrada tanto o serviço primário quanto o secundário, que disponibilizaram as tecnologias duras, como exames de raios $\mathrm{X}$ e baciloscopia. Exames complementares foram solicitados e, por serem realizados em serviços privados, acarretaram custos ao paciente.

O tratamento da maioria dos participantes ocorreu em unidades de saúde da família próximas ao local de residência, sendo realizado por alguns no centro de especialidades por motivo do vínculo ou residirem em área descoberta da ESF. Eles estavam satisfeitos com o tratamento, a acessibilidade e a cordialidade dos profissionais de saúde, mas insatisfeitos com a falta de investigação dos sintomas e por não terem evolução do quadro clínico. Muitos desejavam a inclusão de outros tipos de serviços resolutivos e acesso a outras tecnologias.

Quadro 2 - Necessidade de garantia de acesso a todas as tecnologias que melhorem e prolonguem a vida. Crato, CE, Brasil, 2015

\begin{tabular}{|c|c|}
\hline $\begin{array}{l}\text { Sintomas } \\
\text { respiratórios e } \\
\text { tecnologias } \\
\text { disponibilizadas }\end{array}$ & $\begin{array}{r}\text { Tossia sangue[...] pediram exames do escarro, raio-X, e o daqui [mostra o braço esquerdo-Prova } \\
\text { tuberculínica], [...] esse do nariz [broncoscopia], onde deu a bactéria. (E4) } \\
\text { Uns } 15 \text { dias a } 20 \text { dias começou com uma tosse muito forte. Procurei o posto [ESF] [...] fiz o raio-X, } \\
\text { exame de escarro e comecei o tratamento. (E8) } \\
\text { Vai fazer três anos [...], eu nunca tive febre e tossia pouco. A doutora do posto pediu um raio-X do } \\
\text { tórax, [...] disse: é só uma coisa do passado [...]. O médico[reumatologista] pediu uma tomografia [...] } \\
\text { deu uma extensa lesão e tuberculose. Eu tinha que fazer uma biópsia [...]. Quando eu recebi era } \\
\text { tuberculose. (E9) }\end{array}$ \\
\hline $\begin{array}{l}\text { Porta de } \\
\text { entrada }\end{array}$ & $\begin{array}{c}\text { Eu fui para o hospital, o doutor pediu uma chapa [raio-X] [...] mandou ir para posto de saúde, mostrar } \\
\text { o exame. (E3) } \\
\begin{array}{c}\text { Fui para hospital com tosse e cansaço e lá me internei. [...]. O doutor [pneumologista] me pediu os } \\
\text { exames [...] que mandou ficar pegando os remédios aqui mesmo [ESF]. (E10) }\end{array}\end{array}$ \\
\hline $\begin{array}{l}\text { Satisfação diante } \\
\text { da oferta e dos }\end{array}$ & Considero [satisfeito]. Porque está fazendo o meu tratamento certo. (E5) \\
\hline
\end{tabular}


9 | Oliveira AH, Pinto AGA, Quirino GS, Cruz RSBL, Pereira MLD, Cavalcante EGR

\begin{tabular}{|c|c|}
\hline $\begin{array}{c}\text { recursos } \\
\text { disponíveis nos } \\
\text { serviços de } \\
\text { saúde }\end{array}$ & $\begin{array}{c}\text { Se não fosse esse posto aqui eu estava era morto. (E7) } \\
\text { Nâ. Eue fiquei sozinha [...]. Eu queria mesmo um lugar que resolvesse todos os meus problemas. } \\
\text { (E9) }\end{array}$ \\
\hline
\end{tabular}

Necessidade de ter vínculo com um profissional da equipe

Os participantes apontaram satisfação e vínculo com o enfermeiro durante a consulta e visita domiciliar. Todos os profissionais se preocupavam com o tratamento e aconselhavam quanto ao autocuidado. Quase todos os entrevistados se submeteram aos encaminhamentos e às orientações dos profissionais de saúde. Apenas um participante escolheu o local do tratamento pelo vínculo e pela confiança no médico especialista.

Quadro 3 - Necessidade de ter vínculo com um profissional da equipe. Crato, CE, Brasil, 2015

\begin{tabular}{|c|c|}
\hline $\begin{array}{l}\text { Relação com o } \\
\text { enfermeiro }\end{array}$ & $\begin{array}{c}\text { Ela [enfermeira] me trata muito bem, está sempre sorrindo. Ela realiza visita domiciliar. (E7) } \\
\text { Minha relação com ela é boa, sempre se preocupa em trazer o medicamento, todo mês. (E9) } \\
\text { Sempre muito atenciosa, mas ela não faz visita. (E6) }\end{array}$ \\
\hline $\begin{array}{l}\text { Satisfação com } \\
\text { outros } \\
\text { profissionais }\end{array}$ & $\begin{array}{c}\text { O Agente de Saúde mora aqui próximo, sempre vem aqui em casa e conversa muito comigo } \\
\text { sobre essa doença. (E5) } \\
\text { O médico [pneumologista] [...] sempre me recebeu muito bem. (E4) }\end{array}$ \\
\hline $\begin{array}{l}\text { Protagonismo na } \\
\text { escolha do local de } \\
\text { tratamento }\end{array}$ & $\begin{array}{c}\text { O doutor [enfermeiro], que botou para eu fazer o tratamento perto de onde eu moro, [...] eu } \\
\text { gostei, não gosto de andar muito [para serviços de saúde distantes]. (E3) } \\
\text { Escolhi o posto [de especialidade] porque o doutor [pneumologista] me atende desde quando } \\
\text { eu tive a primeira pneumonia. (E4) }\end{array}$ \\
\hline
\end{tabular}

\section{Necessidade de autonomia e autocuidado na escolha do modo de "andar a vida"}

As orientações e a busca de informações subsidiaram a autonomia e o modo de andar a vida dos participantes, o que ocorreu individualmente com os profissionais de saúde e pela internet - para aqueles que dispunham dessa tecnologia. Um participante relatou não receber nenhuma orientação. Os sentimentos referentes ao modo de andar a vida foram relatados, como satisfação, ânimo e gratidão a "Deus”. Outros relatos discordantes referiram-se a desânimo, incômodo com a autoimagem e dependência dos outros (familiares). 
Quadro 4 - Necessidade de autonomia e autocuidado na escolha do "modo de andar a vida" de pessoas com tuberculose. Crato, CE, Brasil, 2015

\begin{tabular}{|c|r|}
\hline $\begin{array}{c}\text { Orientações sobre } \\
\text { o processo saúde- } \\
\text { doença e fonte de } \\
\text { informação }\end{array}$ & $\begin{array}{r}\text { Ela [enfermeira] sempre diz que eu tinha que me cuidar, se alimentar na hora certa, tomar } \\
\text { remédio. O doutor [pneumologista] também. (E4) }\end{array}$ \\
$\begin{array}{r}\text { Não recebi nenhuma orientação, eu não posso ir ao posto. (E10) } \\
\text { Sentimento } \\
\text { referente ao modo internet. Por curiosidade mesmo, tipo assim, coisas naturais, sucos, vitaminas, } \\
\text { que ajuda na nossa recuperação. (E6) }\end{array}$ \\
$\begin{array}{c}\text { Estou me sentindo aliviada, [...] feliz porque eu estou ficando boa. (E3) } \\
\text { Eu me sinto uma pessoa doente. [...] Eu vivo nas casas dos outros, tenho vontade de } \\
\text { trabalhar e não posso. (E1) }\end{array}$ \\
Eu me sinto um pouco mal[...] a única coisa que me incomoda é a questão do peso que está \\
baixando muito rápido. (E8)
\end{tabular}

\section{Discussão}

No presente estudo, a análise da compreensão das necessidades de saúde das pessoas com TB articula-se com a construção do cuidado. No elemento relativo às necessidades de boas condições de vida, percebeu-se que os participantes apresentaram contradições vivenciadas em seu cotidiano diante de sua condição de vida e trabalho, ao relacionarem saúde à qualidade de vida e fé, enquanto doença esteve associada aos sentimentos de tristeza e enfrentamento.

Os entrevistados reconhecem que a transmissão da TB está relacionada às suas condições de vida, hábitos e moradia quando apontam elementos que podem ter favorecido o adoecimento, mesmo com concepções errôneas ao associá-lo à poluição, ao fumo, dentre outros. As condições de vida e trabalho relacionaram-se à maior concentração de casos de TB em áreas vulneráveis que mantêm os determinantes socioeconômicos na reprodução da doença, como renda, bens de consumo, moradia e aglomerações. ${ }^{9-10}$

Ainda nesse elemento, os participantes apontaram que suas necessidades de saúde, em resposta à $\mathrm{TB}$, demandam por melhores condições socioeconômicas e ambientais, o que 
corresponde à alimentação, à mudança de estilo de vida e ao tratamento. Estudo coreano recomenda que o uso do questionário de percepção breve da doença realizado com pacientes com TB permitiu compreender seus comportamentos e pôde auxiliar os profisionais de saúde no gerenciamento eficaz da doença durante a rotina dos serviços. ${ }^{11}$

Nesse contexto, as barreiras percebidas, como o estado socioeconômico e o uso de álcool, além do sofrimento psicológico, interferem no processo saúde-doença e no tratamento, precisando de intervenções de promoção da saúde. ${ }^{12}$ Assim, considerando que a TB é uma doença socialmente construída, reforça-se a importância para o desenvolvimento de políticas de proteção social que possam reduzi-la. ${ }^{13} \mathrm{Na}$ prática, exigem que os profissionais transcendam a questão biológica e considerem a posição socioeconômica das pessoas com $\mathrm{TB}$, na perspectiva de assegurar o acesso às redes sociais de apoio.

No elemento de necessidade de garantia de acesso a todas as tecnologias que melhorem e prolonguem a vida, os indivíduos relataram confirmação do diagnóstico em tempo inferior a 30 dias. Intervalo menores foram identificados em São José do Rio Preto (SP), com 6 a 30 dias. ${ }^{14}$ Outro estudo, na cidade de Cáli, Colômbia, apontou intervalo de 57 a 72 dias. ${ }^{15}$ Essas diferenças repercutem no controle da doença e demandam a elaboração de indicadores que reforcem o acesso ao diagnóstico e o tratamento imediato, os quais possam ser avaliados pelos serviços de saúde.

Os participantes tiveram acesso aos exames de rotina e outros complementares mais específicos, sendo que esses últimos ocasionaram custos adicionais aos pacientes. Quanto ao ônus, estudos apontam perdas de emprego, gasto em transporte motorizado e na carga econômica, que podem exceder a renda anual das famílias. ${ }^{16}$ Estratégias devem ser elaboradas para garantir o acesso aos exames complementares, considerando a equidade da população vulnerável dentre outras tecnologias leves e leve-duras.

Ainda sobre esse elemento, os participantes realizaram o tratamento em serviços de saúde mais próximos de seus territórios. Para aqueles que foram acompanhados em centro de 
especializados, a escolha ocorreu devido ao vínculo com o profissional de saúde ou por encontrar-se em área descoberta da ESF. Os pacientes tiveram limitado protagonismo na escolha do serviço de saúde, contudo buscaram diferentes pontos de atendimentos, relatando satisfação com o serviço referente ao acesso e ao tratamento.

A insatisfação esteve relacionada à obtenção do diagnóstico tardio ou por ainda apresentar manifestações clínicas. Estudo aponta que o acesso ao diagnóstico e ao tratamento dos casos de TB tem sido realizado especialmente pela atenção primária à saúde, enquanto as fragilidades no manejo dos casos atípicos ocorrem devido ao atraso, erros e necessidade de confirmação diagnóstica. ${ }^{17}$

Estudo avaliativo também apontou a satisfação com o acesso aos serviços primário e secundário. ${ }^{18}$ Assim como na presente pesquisa, a insatisfação também ocorreu em razão da não realização de visita domiciliar por ambos os serviços. ${ }^{18}$ Percebe-se que a acessibilidade universal ocorre pela busca adequada ao controle da TB. Nesse sentido, a superação das deficiências no acesso ao diagnóstico da doença requer uma organização apropriada dos serviços de atenção para atender às necessidades de saúde e assegurar o acesso aos serviços.

No elemento necessidade de ter vínculo com um profissional da equipe, os entrevistados mencionaram que o enfermeiro e outros profissionais de saúde os acolheram durante as consultas, bem como realizaram orientações e visita domiciliar. De modo singular e diferente, um participante revela que as relações com o enfermeiro foram frágeis. Esses mesmos profissionais incentivaram os pacientes para o autocuidado ao longo do tratamento com relação de cuidado e confiança, expressos durante as consultas por meio do zelo, da atenção e do bom relacionamento.

Estudo aponta que o acolhimento dos profissionais de saúde, especialmente na atenção primária, tem fortalecido o vínculo e contribuído para o monitoramento de pessoas com $\mathrm{TB} .{ }^{19} \mathrm{O}$ vínculo e o acolhimento fazem parte da tecnologia leve que pode ser estabelecida e representa ferramentais para otimizar o cuidado aos pacientes. ${ }^{20}$ Diante do exposto, a promoção do cuidado 
13 | Oliveira AH, Pinto AGA, Quirino GS, Cruz RSBL, Pereira MLD, Cavalcante EGR

centrado na pessoa com TB deve ser valorizada na perspectiva de melhorar o processo de trabalho e a qualidade da assistência.

Referente ao último elemento da taxonomia, a necessidade de autonomia e autocuidado na escolha do "modo de andar a vida", identificou-se que os pacientes receberam orientações dos profissionais de saúde referentes aos cuidados com a saúde quanto à alimentação, à adesão ao tratamento e ao retorno das consultas. O acesso à internet foi restrito para a maior parte dos enfermos, que realizaram busca de outros cuidados e informações sobre TB.

De fato, todas as orientações sobre a doença, adesão ao tratamento e necessidade de mudanças no estilo de vida subsidiaram a construção da autonomia dos pacientes, que relataram as ações de saúde que devem seguir. O estímulo à autonomia do paciente e o cuidado centrado no indivíduo apresentam interface com a construção democrática, permitindo, assim, que façam parte das discussões sobre o seu tratamento e plano de cuidado. Contudo, essa prática nas ações de enfermagem apresentou-se desfavorável nos municípios de João Pessoa e Manaus, enquanto em Porto Alegre foi parcialmente favorável. ${ }^{21}$

Dessa forma, a construção compartilhada do conhecimento na busca de autonomia e democracia apresenta-se como caminho possível para a realização de modelos dialógicos e participativos. Assim, a incorporação destas pode contribuir para a relação de confiança entre pacientes-profissionais de saúde e, consequentemente, potencializar os resultados na atenção às pessoas com TB.

Ainda, nesse elemento, o sentimento referente ao modo de andar a vida, os entrevistados expressaram gratidão e estavam incomodados com a autoimagem e a dependência de familiares. As necessidades singulares devem ser reconhecidas pelas equipes de saúde no cuidado, pois, além dos sintomas, têm-se o estigma e o rompimento de atividade de lazer e trabalho. ${ }^{22}$ Ressaltase que a questão da TB deve ser ampliada para fundamentar-se na ética com dignidade, justiça social e acolhimento de pessoas que vivem com condições sociais injustas. ${ }^{23-24}$ Nesse contexto, 
percebe-se a necessidade do envolvimento da equipe de ESF para intervir na busca de cuidado junto à pessoa com $\mathrm{TB}$, na perspectiva de retomar a vida, assim como garantir apoio social e psicológico para auxiliar o processo de enfrentamento do adoecimento.

Como limitação do estudo, aponta-se o número de participantes que impede a generalização dos achados. No entanto, o estudo destaca questões relevantes relacionadas ao acesso à saúde do contexto local, as quais devem ser levadas em consideração pelos gestores e profissionais de saúde.

\section{Conclusão}

$\mathrm{Na}$ análise das necessidades de saúde da pessoa com TB pulmonar, evidenciou-se que a concepção sobre as boas condições de vida encontrava-se articulada às condições do adoecimento refletidas no contexto ambiental em que vivem devido à falta de alimentação e de mudanças de hábitos saudáveis.

Quanto ao acesso a todas as tecnologias que melhorem e prolonguem a vida, enfatizou-se o acesso a tecnologias duras, como a realização dos exames. Relataram satisfação com a acessibilidade aos serviços próximos à sua residência, mas encontravam-se insatisfeitos com a falta do monitoramento dos sintomas e evolução da doença, assim como os custos que tiveram para realizar os exames complementares.

Referente ao vínculo com um profissional da equipe, os pacientes encontravam-se satisfeitos e apontaram ter vínculo, em especial, com os enfermeiros. Salienta-se que a autonomia e o autocuidado na escolha do modo de "andar a vida" foram subsidiados pelas informações e orientações que buscaram quase que exclusivamente com a equipe de saúde.

Diante dos significados e sentidos acerca do processo saúde-doença vivenciados, o enfermeiro e os demais profissionais da equipe de saúde valorizaram a subjetividade e a individualidade da pessoa doente visando ao êxito do processo terapêutico. Embora satisfeitos 
com os recursos de saúde disponíveis, requerem avaliação de saúde e da proteção social, por meio de intervenções do cuidado no âmbito da integralidade, tendo a Atenção Primária como ordenadora da rede de saúde.

Em relação às considerações para a prática da saúde, o estudo aponta que os elementos da taxonomia relacionados à saúde das pessoas com TB podem potencializar o cuidado por meio de estratégias interdisciplinares e intersetoriais que minimizem sofrimentos e considerem suas desigualdades sociais.

Acredita-se ser relevante o desenvolvimento de outras pesquisas que analisem a percepção dos profissionais na assistência sobre a saúde das pessoas em tratamento TB, assim como a descrição das estratégias lançadas durante a assistência para o seu alcance.

\section{Referências}

1.World Health Organization (WHO). Global tuberculosis report 2019. 2019 [Internet]. Geneva: WHO; 2019 [cited 2020 Apr 27]. Available from: https://www.who.int/tb/publications/global_report/en/

2. Bertolozzi MR, Takahashi RF, França FOS, Hino P. A ocorrência da tuberculose e sua relação com as desigualdades sociais: estudo de revisão integrativa na base PubMed. Esc Anna Nery. 2020;24(1):e20180367. doi: 10.1590/2177-9465-ean-2018-0367

3. Maffacciolli R, Oliveira DLLC, Brand EM. Vulnerabilidade e direitos humanos na compreensão de trajetórias de internação por tuberculose. Saúde Soc. 2017;26(1):286-99. doi: 10.1590/S010412902017168038

4. Cecilio LCO, Matsumoto NF. Uma taxonomia operacional de necessidades de saúde. In: Pinheiro R, Ferla AA, Mattos RA, organizadores. Gestão em redes: tecendo os fios da integralidade em saúde [Internet]. Rio de Janeiro: EDUCS; IMS/UERJ: CEPESC; 2006 [acesso em 2020 abr 27]. Cap. 3. Disponível em: https://www.cepesc.org.br/wp-content/uploads/2013/08/GESTA\%CC\%83O-EM-REDES_RS.pdf

5. Temoteo RCA, Carvalho JBL, Lira ALBC, Lima MA, Sousa YG. Enfermagem na adesão ao tratamento da tuberculose e tecnologias em saúde no contexto da atenção primária. Esc Anna Nery. 2019;23(3):e20180321. doi: 10.1590/2177-9465-ean-2018-0321

6. Sicsú AN, Gonzales RIC, Mitano F, Sousa LO, Silva LMC, Ballestero JGA, et al. Práticas de enfermagem centradas no indivíduo com tuberculose: interface com a democracia. Rev Bras Enferm. 
Necessidades de saúde das pessoas com tuberculose pulmonar | 16

2019;72(5):1219-25. doi: 10.1590/0034-7167-2017-0380

7. Goceer S, Gunay O, Ulutabanca RO, Sonkaya ZI. Factors affecting psychosocial adjustments to illness of active tuberculosis patients. Med Sci (Turkey). 2017;6(4):700-5. doi: 10.5455/medscience.2017.06.8644

8. Minayo MC. O desafio do conhecimento: pesquisa qualitativa em saúde. 14ª ed. São Paulo: Hucitec; 2014.

9. Pereira AGL, Escosteguy CC, Valencia LIO, Magalhães MAFM, Medronho RA. Análise espacial de casos de tuberculose e associação com fatores socioeconômicos: uma experiência no município do Rio de Janeiro. Cad Saúde Colet. 2018;26(2):203-10. doi: 10.1590/1414-462X201800020013

10. Pedro AS, Gibson G, Santos JPC, Toledo LM, Sabroza PC, Oliveira RM. Tuberculosis as a maker of inequities in the context of social-spacial transformation. Rev Saúde Pública. 2017;51:9. doi: 10.1590/S1518-8787.2017051006533

11. Min J, Chang Y, Lee KM, Choe KH, An JY. Transcultural adaptation and validation of the Korean version of the brief illness perception questionnaire for patients with pulmonary tuberculosis. J Global Infect Dis. $2017 \quad$ [cited $2020 \quad$ Jun 24];9:113-6. $\quad$ Available from: http://www.jgid.org/text.asp?2017/9/3/113/212583

12. Tola HH, Garmaroudi G, Shojaeizadeh D, Tol A, Yekaninejad MS, Ejeta LT, et al. The effect of psychosocial factors and patients' perception of tuberculosis treatment non-adherence in Addis Ababa, Ethiopia. Ethiop J Health Sci. 2017;27(5):447. doi: 10.4314/ejhs.v27i5.2

13. Moreira ASR, Kritski AL, Carvalho ACC. Determinantes sociais da saúde e custos catastróficos associados ao diagnóstico e tratamento da tuberculose. J Bras Pneumol [Internet]. 2020 [cited 2020 Jun 24];46(5):e20200015. Available from: http://www.jornaldepneumologia.com.br/detalhe_artigo.asp?id=3350

14. Ponce MAZ, Wysocki AD, Arakawa T, Andrade RLP, Vendramini SHF, Sobrinho RAS, et al. Delay in tuberculosis diagnosis in adults in a city of São Paulo State, Brazil, in 2009: a cross-sectional study. Epidemiol Serv Saúde. 2016;25(3):553-62. doi: 10.5123/S1679-49742016000300011

15. Cordoba C, Luna L, Triana DM, Perez F, López L. Factors associated with delays in pulmonary tuberculosis diagnosis and treatment initiation in Cali, Colombia. Rev Panam Salud Pública. 2019;43:e14. doi: 10.26633/RPSP.2019.14

16. Verguet S, Riumallo-Herl C, Gomez GB, Menzies NA, Houben RMGJ, Sumner T, et al. Catastrophic costs potentially averted by tuberculosis control in India and South Africa: a modelling study. Lancet Glob Health. 2017;5(11):e1123-32. doi: 10.1016/S2214-109X(17)30341-8

17. Oliveira AH, Pinto AGA, Lopes MSV, Figueiredo TMRM, Cavalcante EGR. Itinerário terapêutico de pessoas com tuberculose. Esc Anna Nery. 2019;23(3):e20190034. doi: 10.1590/2177-9465-ean-2019-0034

18. Furlan MCR, Marcon SS. Avaliação do acesso ao tratamento de tuberculose sob a perspectiva de 
usuários. Cad Saúde Colet. 2017;25(3):339-47. doi: 10.1590/1414-462X201700030139

19. Ferreira MRL, Santos AA, Orfão NH. O vínculo no tratamento da tuberculose na atenção primária à saúde: uma revisão integrativa. Rev Bras Promoç Saúde. 2019;32:9540. doi:10.5020/18061230.2019.9540

20. Merhy EE, Onocko R, editores. Agir em saúde: um desafio para o público. $2^{a}$ ed. São Paulo: Hucitec; 2002. Em busca de ferramentas analisadoras das tecnologias em saúde: a informação e o dia a dia de um serviço, interrogando e gerindo trabalho em saúde. p. 113-50.

21. Sicsú AN, Gonzales RIC, Mitano F, Sousa LO, Silva LMC, Ballestero JGA, et al. Nursing practices centered on individuals with tuberculosis: an interface with democracy. Rev Bras Enferm. 2019;72(5):1219-25. doi: 10.1590/0034-7167-2017-0380

22. Jung BC, Zillmer JGV, Cunha FTS, Gonzales RIC. Significados das experiências corporais de pessoas com tuberculose pulmonar: a construção de uma nova identidade. Texto Contexto Enferm. 2018;27(2):e2030016. doi: 10.1590/0104-070720180002030016

23. Fortes PD. A justa dose da medida: o tratamento compulsório da tuberculose em questão. Interface (Botucatu). 2016;20(58):743-51. doi: 10.1590/1807-57622015.0775

24. Zoboli E. Autonomia e coerção na justa medida do tratamento da tuberculose. Interface. (Botucatu). 2016;20(58):760-2. doi: 10.1590/1807-57622016.0224

Editora Científica: Tânia Solange Bosi de Souza Magnago

Editora Associada: Graciela Dutra Sehnem

\section{Autor correspondente}

Edilma Gomes Rocha Cavalcante

E-mail: edilma.rocha@yahoo.com.br

Endereço: Rua Pedro Bantin Neto, 525 Crato-CE

CEP:63108-115

\section{Contribuições de Autoria}

\section{1 - Aliéren Honório Oliveira}

Concepção ou desenho do estudo/pesquisa, análise e/ou interpretação dos dados, revisão final com participação crítica e intelectual no manuscrito.

2 - Antonio Germane Alves Pinto

Análise e/ou interpretação dos dados, revisão final com participação crítica e intelectual no manuscrito. 


\section{3 - Glauberto da Silva Quirino}

Análise e/ou interpretação dos dados, revisão final com participação crítica e intelectual no manuscrito.

\section{4 - Rachel de Sá Barreto Luna Callou Cruz}

Análise e/ou interpretação dos dados, revisão final com participação crítica e intelectual no manuscrito.

\section{5 - Maria Lúcia Duarte Pereira}

Análise e/ou interpretação dos dados, revisão final com participação crítica e intelectual no manuscrito.

\section{6 - Edilma Gomes Rocha Cavalcante}

Concepção ou desenho do estudo/pesquisa, análise e/ou interpretação dos dados, revisão final com participação crítica e intelectual no manuscrito.

\section{Como citar este artigo}

Oliveira AH, Pinto AGA, Quirino GS, Cruz RSBL, Pereira MLD, Cavalcante EGR. Necessidades de saúde das pessoas com tuberculose pulmonar. Rev. Enferm. UFSM. 2021 [Acesso em: Ano Mês Dia]; vol.11 e11 1-18. DOI:https://doi.org/10.5902/2179769243901 